\title{
Personality vulnerabilities in adolescent suicidality: The mediating role of psychological distress
}

\author{
Rui C. Campos, PhD \\ Avi Besser, $\mathrm{PhD}$ \\ Helena Abreu, MA candidate \\ Teresa Parreira, MA \\ Sidney J. Blatt, PhD
}

The research literature consistently indicates that self-criticism is related to suicidality. Evidence for the role of dependency, however, is more controversial. This study examines the extent to which these personality vulnerabilities are mediated by psychological distress in the prediction of suicidality. As part of a study of adolescent psychopathology, a sample of 260 Portuguese adolescents (148 [56.9\%] female and 112 [43.1\%] male), ranging in age from 15 to 18 years $(M=16.32, S D=1.19)$ completed measures of personality, suicidal behavior, and current distress, in counterbalanced order. The measures were: self-criticism and dependency from the Depressive Experiences Questionnaire for Adolescents; two psychological distress scales, social withdrawal from the Youth Self Report and depression from the Center for Epidemiologic Studies of Depression Scale; and a measure of suicidality from the Suicide Behaviors Questionnaire Revised. Structural equation modeling indicated that self-criticism and dependency were both significantly associated with suicidality. Psychological distress, however, as measured by withdrawal and depression, fully mediated these relationships, but did not moderate them. The authors conclude that adolescents with higher levels

\footnotetext{
This article was acepted under the editorship of Dr. W. Walter Menninger.

Rui C. Campos is in the Department of Psychology and Center for Research on Education and Psychology (CIEP), University of Évora, Portugal; Avi Besser is in the Department of Behavioral Sciences and the Center for Research in Personality, Life Transitions, and Stressful Life Events, Sapir Academic College, D. N. Hof Ashkelon, Israel; Helena Abreu and Teresa Parreira are at the University of Evora, Portugal; Sidney J. Blatt is professor emeritus, Yale University, New Haven, Connecticut. We would like to acknowledge all of the participants in this study. Correspondence may be sent to Rui C. Campos, Department of Psychology, University of Évora, Apartado 94, Évora, Portugal 7002-554; e-mail: rcampos@uevora.pt (Copyright (C) 2014 The Menninger Foundation)
} 
Campos et al.

of self-criticism and dependency are at greater risk for experiencing intense psychological distress - high levels of social withdrawal and depression-that account for their vulnerability to suicide risk.(Bulletin of the Menninger Clinic, 78[2], 115-139)

The understanding and treatment of depression has changed its focus from symptomatic expressions of depression to an understanding of the vulnerabilities to depression. Several psychologically oriented investigators of depression (Arieti \& Bemporad, 1980; Beck, 1983; Blatt, 1974, 2004; Blatt, Quinlan, Chevron, McDonald, \& Zuroff, 1982; Bowlby, 1980) have identified two fundamental vulnerabilities associated with depression: disturbances in interpersonal relationships, and disruptions in the sense of self. Individuals with these vulnerabilities have different early life experiences and respond to differing stressful events and therapeutic interventions (see Blatt, 2004). Blatt (1974; Blatt et al., 1982) has labeled the vulnerability associated with interpersonal relatedness as anaclitic or dependent and that associated with the sense of self as introjective or self-critical.

Dependency and self-criticism, two primary sources of vulnerability (e.g., Blatt, 2004; Luyten \& Blatt, 2011; Luyten, Corveleyn, $\&$ Blatt, 2005), are in fact part of a broad conceptualization of personality development and psychopathology (e.g., Blatt, 2008) that demonstrates how the development of a positive and integrated sense of identity and the capacity to establish and maintain mature, reciprocal, and satisfying interpersonal relationships are central components of development that evolve synergistically throughout life, from infancy to senescence (Blatt, 2008). Differences in the relative emphasis placed on processes of relatedness and self-definition delineate two fundamental personality stylesdependent and self-critical-that cause individuals to engage in and experience life differently (Besser \& Priel, 2003, 2005, 2010, 2011; Blatt, 2008; Blatt et al., 1982; Blatt \& Zuroff, 1992).

Research indicates that self-critical vulnerability can play an important role in suicide (Beck 1983; Blatt, 1974, 1995; Blatt et al., 1982; Fazaa \& Page, 2006, but findings are more equivocal about interpersonal vulnerability. Some studies (Blatt et al., 1982; Fazaa \& Page, 2006) indicate that the suicidal activity of anaclitic or dependent individuals is often carried out in a manner that is 